\title{
Valproic acid for children below 2 years of age with epilepsy
}

Osama Y. Muthaffar, MBBS, SBPN, Salha M. Almahmudi, PharmD, Muna O. Alrabghi, PharmD, Maria M. Bin Mahfouz, PharmD, Nuha S. Alfawaz, PharmD.

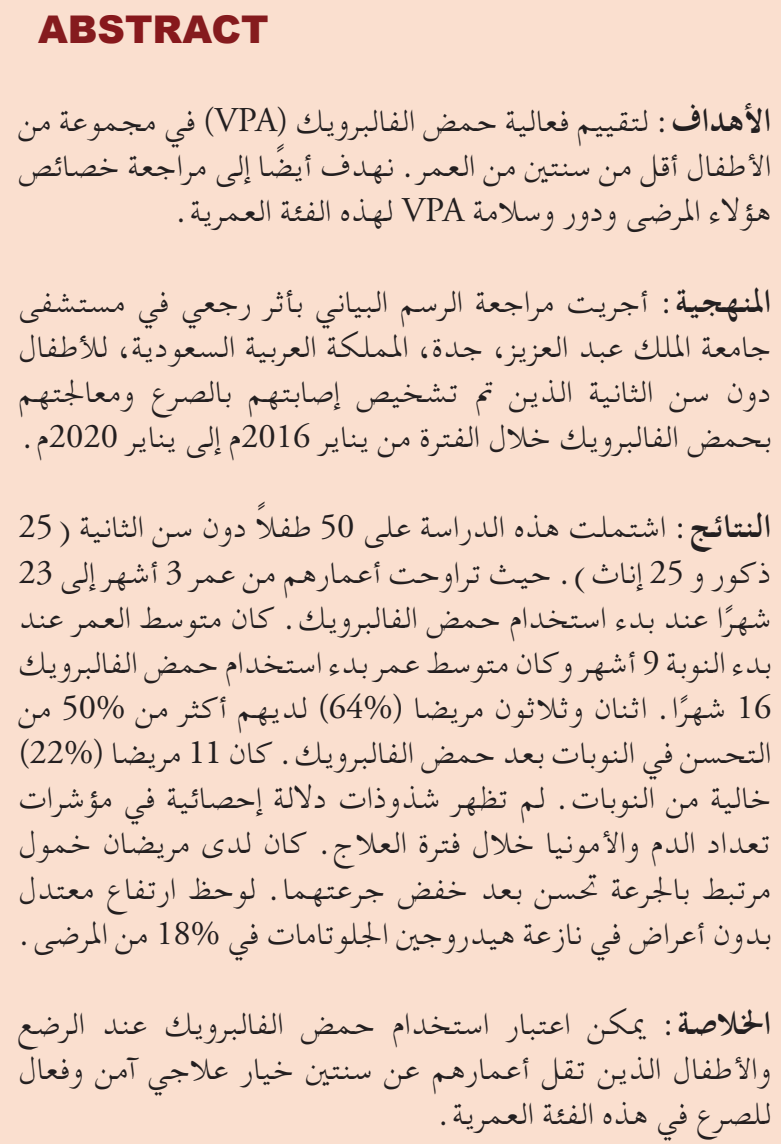

Objectives: To evaluate the efficacy of valproic acid (VPA) in a cohort of children below 2 years of age. We also aim to review the characteristics of such patients and the role and safety of VPA for this age group.

Methods: A retrospective chart review conducted at King Abdulaziz University Hospital, Jeddah, Kingdome of Saudi Arabia, for children below 2 years of age diagnosed with epilepsy and treated with valproic acid from January 2016 to January 2020.

Results: The cohort for this study includes 50 children below the age of 2 years ( 25 males, 25 females). Aged 3 months to 23 months at commencing valproic acid. The mean age of seizure onset was 9 months and the mean age of starting valproic acid was 16 months. Thirty-two patients (64\%) had more than $50 \%$ seizure improvement after valproic acid. Eleven patients (22\%) were seizure-free. No statistical significance abnormalities in blood count indices and ammonia were seen during the treatment period. Two patients had dose-related lethargy that improved after decreasing their dosage. Asymptomatic mild elevation in glutamate dehydrogenase was noticed in $18 \%$ of patients.

Conclusion: Using valproic acid in infants and children below the age of 2 years can be considered as a safe and effective treatment option for epilepsy in this age group.

Neurosciences 2021; Vol. 26 (4): 357-365 doi: 10.17712/nsj.2021.4.20210075

From the Department of Pediatrics (Muthaffar), and from the Faculty of Pharmacy (Almahmudi, Alrabghi, Bin Mahfouz, Alfawaz), King Abdulaziz University, Jeddah, Kingdom of Saudi Arabia

Received 25th May 2021. Accepted 25th August 2021.

Address correspondence and reprint request to: Dr. Osama Y. Muthaffar, Department of Pediatrics, King Abdulaziz University, Jeddah, Saudi Arabia, Jeddah, Kingdom of Saudi Arabia.E-mail: oymuthaffar@kau. edu.sa. ORCID ID: https://orcid.org/0000-0002-3458-1697

E pilepsy is a major neurological disorder that occurs across different age groups. Children below the age of 2 years are an important subcategory. They are more prone to have seizures compared to older children. ${ }^{1}$ Moreover, uncontrolled seizures in early life can result in poor outcomes on cognition, motor and language development. ${ }^{2}$ Seizure-freedom in children below 2 years ranges between $49-57 \%$ compared to $>60 \%$ in older children. ${ }^{3,4} \mathrm{~A}$ myriad group of etiologies can result in seizures in this vulnerable age group including: electrolytes disturbances, asphyxia, inborn errors of metabolism, structural brain etiologies, infection and genetic etiologies. Brain ion channels, neurotransmitters and cellular maturation is unique in this age group 
making them at higher risk for seizures. Response to antiseizure medications (ASMs) also is different in this category of patients. ${ }^{5}$ Safety and options of ASMs is also another challenge.

Multiple electroclinical epilepsy syndromes occur in this category like West syndrome, Dravet syndrome and myoclonic epilepsy which can be challenging to treat. Most available ASMs has not been studied in this age group. Valproic acid (VPA) has been available as a treatment for more than 50 years. It is classified as broad spectrum ASM. It is one of old generation ASM with few side effects reported. ${ }^{6}$ Its use below the age of 2 years is limited by its potential hepatotoxicity especially when used as polytherapy or in high dosage. Other factors like POLG mutation and mitochondrial disease could increase the chance for hepatic insult. ${ }^{7}$

The incidence of hepatotoxicity in children in general is higher than adults, 1:5000 compared to 1:40000, respectively. Multiple proposed mechanisms have been implicated like reduction of intracellular CoA, defects of oxidative phosphorylation and inhibition of gluconeogenesis. ${ }^{89}$

The aim of this study is to evaluate the efficacy and safety of valproic acid (VPA) in this age group and to review the clinical characteristics of this cohort. Few studies in literature evaluated VPA usage below the age of 2 years. ${ }^{7,9}$

Methods. Study setting. This is a retrospective chart review study. The data were collected from the paper records and electronic medical records at King Abdulaziz University Hospital, Jeddah, Kingdome of Saudi Arabia. Files of patients from January 2016 to January 2020 were reviewed. The study protocol was approved by the institutional review board of King Abdulaziz University (311-20). The inclusion criteria of the study were children treated with VPA below the age of two years, diagnosed with epilepsy, children without liver disease or metabolic conditions affecting the liver and on VPA for a duration $\geq 3$ months. Children above 2 years old, with liver disease or used VPA less than 3 months were excluded. The primary outcome measures were to describe different clinical manifestations of those children and evaluate efficacy and safety of VPA.

Demographic data, VPA dose and duration, etiologies, the number of current and previous

Disclosure. Authors have no conflict of interests, and the work was not supported or funded by any drug company. antiseizure medications, seizure improvement, side effects and blood counts were reviewed and recorded. Patient consents were waived as the data were collected anonymously from medical records.

Statistical analysis. The mean and standard deviation were used to describe variables like age and number of medications. Percentages were also used to describe qualitative data like sex and seizure improvement. Association test statistics were calculated to examine the seizure improvement on study groups of pediatric patients. Data compilation and analyses were conducted using IBM statistical package for the social sciences version for Windows, version 21 (IBM Corp., Armonk, N.Y., USA). We evaluated associations as likelihood ratio Tests (LR), $p$-value (Siq), confidence interval (95\%), and chi-square tests. A p-value less than 0.05 was considered to be statistically significant.

Results. Demographic characteristics of the participants. The study included 50 children below the age of 2 years on VPA treatment. Of the 50 patients, $25(50 \%)$ males and $25(50 \%)$ females (Table 1). The mean age of infants and children from 3 months to 24 months started on VPA was 16 months. Valproic acid average dose was $31.4 \mathrm{mg} / \mathrm{kg} /$ day among this cohort. The average duration of VPA usage was 14 months.

Clinical diagnosis, neuroimaging and EEG features. Symptomatic etiologies were identified in $88 \%$ of patients like structural etiologies, genetic causes and asphyxia. Generalized epileptic discharges on EEG were documented in 21 patients (42\%). Focal epileptic discharges represented in 8 patients (16\%). Infantile spasms and hypsarrhythmia on EEG were seen in 7 patients (14\%). Non-specific slow EEG background and encephalopathy were in 14 children (28\%). Most of the included children has developmental delay (82\%). Clinical and diagnostic investigations are summarized in Table 2.

VPA effect and side effects. In all the included infants and children, VPA was used as a second line ASM. The initial total number of ASMs prescribed for all

Table 1 - Demographic parameters of study participants.

\begin{tabular}{lcc}
\hline Parameter & Mean & SD \\
\hline Age & 3.43 (years) & 1.71 \\
Age of seizure onset & $9.57(\mathrm{~m})$ & 5.83 \\
Age of VPA start & $16.29(\mathrm{~m})$ & 4.87 \\
Duration of VPA & $14.86(\mathrm{~m})$ & 15.50 \\
VPA Dose & $31.4(\mathrm{mg} / \mathrm{kg} /$ day) & 7.4 \\
\hline \multicolumn{2}{c}{ SD - Standard deviation, M - months } \\
\hline
\end{tabular}


Table 2 - Clinical characteristics.

\begin{tabular}{|c|c|c|c|}
\hline Patient / Clinical diagnosis & EEG & Brain imaging & Development \\
\hline Microcephaly & Focal discharges & Normal & Cognitive delay \\
\hline Focal epilepsy & Focal discharges & Lissencephaly & $\mathrm{GDD}^{*}$ \\
\hline Meningitis (HSV meningitis) & $\begin{array}{l}\text { Generalized epileptic } \\
\text { discharges }\end{array}$ & Atrophy & GDD \\
\hline Focal epilepsy & Focal discharges & Normal & Normal \\
\hline Focal epilepsy & Focal discharges & Sturge weber syndrome & Mild hemiparesis \\
\hline $\begin{array}{c}\text { Infantile spasms, congenital Muscular } \\
\text { dystrophy }\end{array}$ & Hypsarrhythmia & Hydrocephalus, Muscle eye brain disease & GDD \\
\hline Hydrocephalus & Slow background & Hydrocephalus and brain tumor (astrocytoma) & GDD \\
\hline Noonan syndrome and infantile spasms & Hypsarrhythmia & Normal & GDD \\
\hline $\begin{array}{l}\text { Epilepsy (genetic), infantile spasms, } \\
\text { panhypopituitarism }\end{array}$ & Hypsarrhythmia & $\begin{array}{c}\text { brain atrophy \& corpus callosum agenesis also } \\
\text { panhypopituraism }\end{array}$ & GDD \\
\hline Meningoencephalitis & $\begin{array}{l}\text { Generalized epileptic } \\
\text { discharges }\end{array}$ & Atrophy and cystic encephalomalacia & Normal \\
\hline VP shunt hydrocephalus & $\begin{array}{l}\text { Generalized epileptic } \\
\text { discharges }\end{array}$ & Hydrocephalus \& Chiari malformation type 2 & GDD \\
\hline VP shunt Hydrocephalus & $\begin{array}{l}\text { Generalized epileptic } \\
\text { discharges }\end{array}$ & Hydrocephalus & GDD \\
\hline Metabolic, aminoacidopathy & Encephalopathy & Normal & Normal \\
\hline $\begin{array}{c}\text { Down syndrome } \& \text { congenital heart } \\
\text { disease }\end{array}$ & Focal epileptic discharges & Enlarged ventricles & Cognitive delay \\
\hline Focal seizures & Slow background & Normal & Normal \\
\hline GM1 gangliosidosis & Slow background & Brain atrophy & GDD \\
\hline Meningoencephalitis & $\begin{array}{l}\text { Generalized epileptic } \\
\text { discharges }\end{array}$ & Normal & Normal \\
\hline Mitochondrial disease & Encephalopathy & Mitochondrial disease (leigh disease) & GDD \\
\hline Idiopathic epilepsy & Slow background & Normal & Normal \\
\hline Infantile spasms & Hypsarrhythmia & Hypoxic ischemic encephalopathy & GDD \\
\hline Birth asphyxia & $\begin{array}{l}\text { Generalized epileptic } \\
\text { discharges }\end{array}$ & Periventricular leukomalacia & GDD \\
\hline $\mathrm{NCL}^{*}$ type 14 & $\begin{array}{l}\text { Generalized epileptic } \\
\text { discharges }\end{array}$ & Brain atrophy & GDD \\
\hline Down syndrome & Encephalopathy & Hypoxic ischemic encephalopathy & GDD \\
\hline $\begin{array}{l}\text { Birth asphyxia. Lennox-Gastaut } \\
\text { syndrome }\end{array}$ & $\begin{array}{l}\text { Generalized epileptic } \\
\text { discharges }\end{array}$ & $\begin{array}{l}\text { Hypoxic ischemic encephalopathy } \& \text { brain } \\
\text { atrophy }\end{array}$ & GDD \\
\hline Idiopathic epilepsy & Encephalopathy & Normal & Normal \\
\hline $\begin{array}{c}\text { Down syndrome and generalized } \\
\text { epilepsy }\end{array}$ & Slow background & Normal & GDD \\
\hline $\begin{array}{l}\text { Wolf-Hirschhorn syndrome } \\
\text { (chromosome 4P deletion), hypotonia, } \\
\text { meningitis, myoclonic epilepsy }\end{array}$ & $\begin{array}{l}\text { Generalized epileptic } \\
\text { discharges }\end{array}$ & Meningitis and agenesis of corpus callosum & GDD \\
\hline $\begin{array}{l}\text { Birth asphyxia. Lennox-Gastaut } \\
\text { syndrome }\end{array}$ & $\begin{array}{c}\text { Generalized epileptic } \\
\text { discharges }\end{array}$ & Occipital encephalomalacia & GDD \\
\hline Brain tumor & Focal discharges & Brain tumor (Dysembryoplastic neuroepithelial) & Normal \\
\hline
\end{tabular}

patients was 141 (mean 2.8, median 3). The number of ASM after starting VPA and 3 months of follow up was 94 (mean 1.8, median 2) (antiseizure medications list in Table 3). Valproic acid dosage mean was 31.4 $\mathrm{mg} / \mathrm{kg} /$ day. After starting VPA, 32 patients (64\%) showed more than $50 \%$ seizure improvement (11 of them were seizure free). Eighteen patients (36\%) had less than $50 \%$ seizure improvement (5 of them had no major improvement in their baseline seizures.

Two patients showed signs of encephalopathy: sleepiness and hypoactivity. Valproic acid dose decrement led to clinical improvement. Complete blood counts were carried out before and after VPA. No major changes were seen in white blood cells or platelets. 
Table 2 - Clinical characteristics.

\begin{tabular}{|c|c|c|c|}
\hline Patient/ Clinical diagnosis & EEG & Brain imaging & Development \\
\hline Stroke and epilepsy & $\begin{array}{l}\text { Generalized epileptic } \\
\text { discharges }\end{array}$ & infarction, watershed, bilateral parietal occipital & GDD \\
\hline $\begin{array}{l}\text { Infantile spasms, Down syndrome, } \\
\text { congenital heart disease }\end{array}$ & Hypsarrhythmia & Normal & GDD \\
\hline Dravet syndrome & $\begin{array}{l}\text { Generalized epileptic } \\
\text { discharges }\end{array}$ & Atrophy and encephalomalacia post meningitis & GDD \\
\hline $\begin{array}{l}\text { Birth asphyxia. Lennox-Gastaut } \\
\text { syndrome }\end{array}$ & $\begin{array}{l}\text { Generalized epileptic } \\
\text { discharges }\end{array}$ & Severe atrophy and hypoxia related changes & GDD \\
\hline Birth asphyxia & $\begin{array}{l}\text { Generalized epileptic } \\
\text { discharges }\end{array}$ & Severe atrophy and hypoxia related changes & GDD \\
\hline $\begin{array}{l}\text { Neurometabolic disease, Dandy-Walker } \\
\text { malformation, global developmental } \\
\text { delay }\end{array}$ & $\begin{array}{l}\text { Generalized epileptic } \\
\text { discharges \& } \\
\text { encephalopathy }\end{array}$ & Dandy-Walker malformation and brain atrophy & GDD \\
\hline Focal epilepsy & Temporal discharges & Normal & Normal \\
\hline $\begin{array}{l}\text { Propionic acidemia, neurodegenerative } \\
\text { brain disorder }\end{array}$ & Encephalopathy & Atrophy & GDD \\
\hline Neurodegenerative brain disorder & Encephalopathy & Atrophy, white matter abnormalities & GDD \\
\hline $\begin{array}{l}\text { Hydrocephalus, Arnold- } \\
\text { Chiari malformation, MMC } \\
\text { (Myelomeningocele) }\end{array}$ & Encephalopathy & Hydrocephalus, Arnold-Chiari malformation & GDD \\
\hline $\begin{array}{l}\text { Chromosomal } 18 \mathrm{q}-\text { syndrom, multiple } \\
\text { congenital anomalies }\end{array}$ & $\begin{array}{l}\text { Generalized epileptic } \\
\text { discharges \& } \\
\text { encephalopathy }\end{array}$ & $\begin{array}{l}\text { Periventricular white matter volume loss, corpus } \\
\text { callosum hypoplasia }\end{array}$ & GDD \\
\hline Infantile spasms, hyperglycinemia & Hypsarrhythmia & brain atrophy, hypoplastic corpus callosum & GDD \\
\hline $\begin{array}{l}\text { Dysmorphic features, caudal regression } \\
\text { syndrome }\end{array}$ & $\begin{array}{l}\text { Generalized epileptic } \\
\text { discharges }\end{array}$ & White matter volume loss & GDD \\
\hline $\begin{array}{l}\text { Neurodegenerative brain disease, } \\
\text { progression of milestones, } \\
\text { hypopituitarism }\end{array}$ & Focal discharges & Hydrocephalus & GDD \\
\hline Aicardi syndrome & Hypsarrhythmia & hypoplastic corpus callosum & GDD \\
\hline $\mathrm{NCL}$ & $\begin{array}{l}\text { Generalized epileptic } \\
\text { discharges }\end{array}$ & Brain atrophy & GDD \\
\hline Down syndrome, birth asphyxia & $\begin{array}{l}\text { Generalized epileptic } \\
\text { discharges \& } \\
\text { encephalopathy }\end{array}$ & Hypoxia changes & GDD \\
\hline Preterm, birth asphyxia & $\begin{array}{l}\text { Generalized epileptic } \\
\text { discharges \& } \\
\text { encephalopathy }\end{array}$ & Brain atrophy & GDD \\
\hline Birth asphyxia & Encephalopathy & Brain atrophy & GDD \\
\hline $\begin{array}{l}\text { Neurometabolic disease, glutamine } \\
\text { synthase deficiency }\end{array}$ & Encephalopathy & Colpocephaly with dilation of lateral ventricles & GDD \\
\hline Idiopathic epilepsy & $\begin{array}{l}\text { Generalized epileptic } \\
\text { discharges }\end{array}$ & Normal & GDD \\
\hline
\end{tabular}

Comparing changes in white blood cells before and after VPA usage showed a statistically insignificant $p$-value $(p=0.747)$. Similarly, for platelets $p$-value before and after treatment was not statistically significant $(p=0.585)$ (Table 4). Valproic acid levels were taken after 3 months of treatment and repeated if needed to gauge drug dosage. Liver function tests (LFTs) including aspartate aminotransferase (AST), alanine aminotransferase (ALT), glutamate dehydrogenase (GGT), alkaline phosphatase (ALP) and total bilirubin were done before and 3 months or more after VPA (Table 5). A mild increase in ALT, GGT and ALP of less than 2 times the normal was seen in patients on polytherapy (3 or more ASM) and patients on another enzyme-inducing medication (carbamazepine). Abnormal LFTs were statistically significant when comparing before and after VPA usage (ALT $p=0.002$, GGT $p=0.013$ and ALP $p=0.002)$. Ammonia level was also reviewed before and 
Table 3 - Antiseizures medications.

\begin{tabular}{|c|c|}
\hline Patient / ASMs before & ASMs after VPA \\
\hline 1 (Levetiracetam) & 1 (Valproic acid) \\
\hline 1 (Levetiracetam) & 2 (Valproic acid, Levetiracetam) \\
\hline 6 (Topiramate, Carbamazepine, Levetiracetam, Lamotrigine, Prednisolone, IVIG) & 2 (Valproic acid, Lacosamide) \\
\hline 5 (Prednisolone, Topiramate, Carbamazepine, Levetiracetam, Lamotrigine) & 2 (Valproic acid, Topiramate) \\
\hline 4 (Carbamazepine, Levetiracetam, Lamotrigine, Topiramate) & 3 (Carbamazepine, Levetiracetam, Valproic acid) \\
\hline 2 (Vigabatrin, Prednisolone) & 2 (Vigabatrin, Valproic acid) \\
\hline 2 (Levetiracetam, Phenytoin) & 2 (Levetiracetam, Valproic acid) \\
\hline 2 (Vigabatrin, Prednisolone) & 1 (Valproic acid) \\
\hline 3 (Levetiracetam, Vigabatrin, Prednisolone) & 2 (Valproic acid, Lamotrigine) \\
\hline 3 (Levetiracetam, Clonazepam, Topiramate) & 2 (Valproic acid, Levetiracetam) \\
\hline 3 (Levetiracetam, Carbamazepine, Topiramate) & 2 (Valproic acid, Levetiracetam) \\
\hline 1 (Levetiracetam) & 1 (Valproic acid)) \\
\hline 5 (Clonazepam, Levetiracetam, Phenobarbital, Carbamazepine Topiramate) & 2 (Clonazepam, Valproic acid) \\
\hline 2 (Levetiracetam, Topiramate) & 1 (Valproic acid) \\
\hline 1 (Levetiracetam) & 1 (Valproic acid) \\
\hline 5 (Phenytoin, Levetiracetam, Phenobarbital, Topiramate, Carbamazepine) & 3 (Valproic acid, Levetiracetam, Lamotrigine) \\
\hline 2 (Levetiracetam, Topiramate) & 1 (Valproic acid) \\
\hline 3 (Levetiracetam, Phenobarbital, Topiramate) & 2 (Levetiracetam, Valproic acid) \\
\hline 3 (Levetiracetam, Phenytoin, Topiramate) & 2 (Levetiracetam, Valproic acid) \\
\hline 3 (Topiramate, Levetiracetam, Prednisolone) & 3 (Clonazepam, Valproic acid, Lamotrigine) \\
\hline 2 (Levetiracetam, Clonazepam) & 2 (Levetiracetam, Valproic acid) \\
\hline 3 (Carbamazepine, Topiramate, Clonazepam) & 3 (Topiramate, Levetiracetam, Valproic acid) \\
\hline 1 (Levetiracetam) & 1 (Valproic acid) \\
\hline 6 (Prednisolone, Vigabatrin, Levetiracetam, Topiramate, Lamotrigine, Clonazepam) & 3 (Levetiracetam, Clonazepam, Topiramate) \\
\hline 3 (Levetiracetam, Phenytoin, Topiramate) & 1 (Valproic acid) \\
\hline 1 (Levetiracetam) & 1 (Valproic acid) \\
\hline 1 (Phenobarbital) & 1 (Valproic acid) \\
\hline 1 (Levetiracetam) & 1 (Valproic acid) \\
\hline 2 (Levetiracetam, Carbamazepine) & 1 (Valproic acid) \\
\hline 2 (Levetiracetam, Carbamazepine) & 3 (Levetiracetam, Lamotrigine, Valproic acid) \\
\hline 3 (Vigabatrin, Topiramate, Levetiracetam, Prednisolone) & 2 (Vigabatrin, Valproic acid) \\
\hline 6 (Levetiracetam, Lamotrigine, Topiramate, Prednisolone, Clonazepam, Phenytoin) & 3 (Levetiracetam, Lamotrigine, Valproic acid) \\
\hline 3 (Leveritacetam, topiramate, phenobarbital) & 2 (Levetiracetam, Valproic acid) \\
\hline 4 (Leveritacetam, Topiramate, Phenobarbital, Prednisolone) & 2 (Levetiracetam, valproic acid) \\
\hline 4 (Levetiracetam, Topiramate, Carbamazepine, Pyridoxine) & 3 (Levetiracetam, Valproic acid, Topiramate) \\
\hline 2 (Levetiracetam, Topiramate) & 1 (Valproic acid) \\
\hline 2 (Levetiracetam, Carbamazepine) & 1 (Valproic acid) \\
\hline
\end{tabular}

after treatment. No statistically significant changes were observed ( $p=0.8$ ) (Table 6).

Discussion. This study evaluated the use of valproic acid in children below the age of 2 years. No previous similar studies were performed in the Middle East as far as we know. Valproic acid has been used safely in different age groups for decades. It acts on GABA $(\gamma$ aminobutyric acid) in the central nervous system and blocks voltage-gated ion channels "sodium, potassium, and calcium". ${ }^{10,11}$ Reports of acute drug reaction and hepatotoxicity were more common below the age of two years. ${ }^{12,13,14}$ In our study, no reported death related to VPA. Multiple studies showed the safety of valproic acid as monotherapy in younger patients and less side effects were reported. ${ }^{15-18}$ We noticed less ASMs (94 medications) compared to (141) before starting VPA. This can be explained by the improvement in seizures burden noticed in $64 \%$ of the cohort that led to weaning-off other ASMs. 
Table 3 - Antiseizures medications.

\begin{tabular}{|c|c|c|}
\hline Patients & ASMs before & ASMs after VPA \\
\hline & $\begin{array}{c}5 \text { (Levetiracetam, Topiramate, Carbamazepine, } \\
\text { Phenobarbital, Vigabatrin) }\end{array}$ & 2 (Topiramate, Valproic acid) \\
\hline & 1 (Levetiracetam) & 1 (Valproic acid) \\
\hline & $\begin{array}{c}4 \text { (Levetiracetam, Phenytoin, Phenobarbital, } \\
\text { Topiramate) }\end{array}$ & 3 (Levetiracetam, Valproic acid) \\
\hline & 3 (Prednisolone, Vigabatrin, Lamotrigine) & 3 (Valproic acid, Levetiracetam, Clonazepam) \\
\hline & 2 (Levetiracetam, Carbamazepine) & 1 (Valproic acid) \\
\hline & 3 (Levetiracetam, Carbamazepine, Topiramate) & 2 (Levetiracetam, Valproic acid) \\
\hline & $\begin{array}{c}4 \text { (Vigabatrin, Prednisolone, Levetiracetam, } \\
\text { Lamotrigine) }\end{array}$ & 3 (Valproic acid, Clonazepam, Topiramate) \\
\hline & 3 (Levetiracetam, Carbamazepine, Topiramate) & 2 (Valproic acid, Clonazepam) \\
\hline & 3 (Levetiracetam, Topiramate, Carbamazepine) & 2 (Valproic acid, Prednisolone) \\
\hline & 3 (Levetiracetam, Topiramate, Valproic acid) & 2 (Valproic acid, Topiramate) \\
\hline & 2 (Levetiracetam, Lamotrigine) & 1 (Valproic acid) \\
\hline & $\begin{array}{c}4 \text { (Phenobarbital, Lamotrigine, Levetiracetam, } \\
\text { Topiramate) }\end{array}$ & 2 (Levetiracetam, valproic acid) \\
\hline & 1 (Topiramate) & 2 (Levetiracetam, Valproic acid) \\
\hline Total ASMs & 141 & 94 \\
\hline Mean & 2.8 & 1.8 \\
\hline Median & 3 & 2 \\
\hline
\end{tabular}

Table 5 - Liver function tests before and after VPA.

\begin{tabular}{|c|c|c|c|c|c|}
\hline $\begin{array}{l}\text { Liver Function Test (Before VPA doses } \\
\text { received) }\end{array}$ & AST & ALT & GGT & ALP & Birlli(Total) \\
\hline & & & $\mathrm{n}(\%)$ & & \\
\hline Normal & $32(64)$ & $45(90)$ & $44(88)$ & $40(80)$ & $50(100)$ \\
\hline Low & 0 & $3(6)$ & $2(4)$ & $7(14)$ & $(0)$ \\
\hline Abnormal (High) & $18(36)$ & $2(4)$ & $4(8)$ & $3(6)$ & $(0)$ \\
\hline $\mathrm{M} \pm \mathrm{SD}$ & $37.8 \pm 18.98$ & $33.28 \pm 29.7$ & $36.7 \pm 42.6$ & $236.5 \pm 76.9$ & $3.12 \pm 1.12$ \\
\hline \multicolumn{6}{|c|}{ Liver Function Test (After VPA doses received) } \\
\hline Normal Range & $28(56)$ & $44(88)$ & $36(72)$ & $39(78)$ & $50(100)$ \\
\hline Low & $2(4)$ & $4(8)$ & $5(10)$ & $9(18)$ & 0 \\
\hline Abnormal (High) & $20(40)$ & $2(4)$ & $9(18)$ & $2(4)$ & 0 \\
\hline $\mathrm{M} \pm \mathrm{SD}$ & $38 \pm 20.13$ & $26.24 \pm 15.9$ & $46.58 \pm 67.5$ & $233 \pm 86.8$ & $2.62 \pm 1.21$ \\
\hline $\begin{array}{l}P \text {-value Sig. (2-tailed) (comparison } \\
\text { between each of subcategory of LFTs) }\end{array}$ & $F(1.076)=0.435$ & $\mathrm{~F}(3.372)=0.002$ & $F(2.897)=0.013$ & $\mathrm{~F}(9.378)=0.002$ & $F(1.011)=0.423$ \\
\hline
\end{tabular}

Liver function enzymes abnormalities were seen in patients on 3 or more ASMs or on enzyme-inducing medications. Compared to other studies, 0 to $50 \%$ of individuals on VPA can develop asymptomatic elevation in LFTs. ${ }^{19-21}$ Asymptomatic mild elevation in GGT was noticed in $18 \%$ of patients of the cohort. ALT and ALP changes are likely because some results were lower than normal were included. International Serious Adverse Events Consortium (iSAEC) defined drug-induced liver injury (DILI) criteria by Aithal et al. ${ }^{22,23}$ None of our cohort met the criteria of DILI. In our cohort, $72 \%$ had asymptomatic hyperammonemia which is statistically not significant $(p=0.8)$ when comparing before and after treatment with VPA. Hyperammonemia is one of the common side effects of VPA. Some studies found 16 to $100 \%$ asymptomatic hyperammonemia in individuals taking VPA. ${ }^{24,25}$

Valproic acid side effects can be related to its dosage. ${ }^{26,27}$ Bone marrow suppression, pancreatitis, hepatotoxicity and acute encephalopathy were related to high dose of VPA in some cases (more than $40 \mathrm{mg} /$ 
Table 4 - Complete blood counts before and after VPA (White blood cells (WBCs) and platelets, PLT).

\begin{tabular}{|c|c|c|c|c|}
\hline \multirow[t]{2}{*}{ Patient } & \multicolumn{2}{|c|}{ WBCs } & \multicolumn{2}{|c|}{ PLT } \\
\hline & Before & After & Before & After \\
\hline & 15.56 & 13.91 & 405 & 309 \\
\hline & 12.05 & 11 & 418 & 388 \\
\hline & 8 & 7 & 177 & 201 \\
\hline & 13.56 & 8.15 & 953 & 616 \\
\hline & 18.25 & 9 & 444 & 267 \\
\hline & 10.5 & 12 & 319 & 314 \\
\hline & 5.77 & 9.12 & 382 & 645 \\
\hline & 6.6 & 11.4 & 222 & 418 \\
\hline & 4.3 & 12 & 367 & 190 \\
\hline & 6.12 & 8.18 & 154 & 507 \\
\hline & 8 & 8 & 500 & 350 \\
\hline & 9 & 12 & 167 & 269 \\
\hline & 13.90 & 10 & 389 & 336 \\
\hline & 20 & 9 & 412 & 230 \\
\hline & 7 & 9.5 & 228 & 394 \\
\hline & 13 & 12.5 & 241 & 290 \\
\hline & 8 & 8 & 405 & 430 \\
\hline & 9.5 & 11 & 306 & 351 \\
\hline & 12.6 & 10.2 & 260 & 387 \\
\hline & 11 & 13 & 355 & 329 \\
\hline & 13 & 9 & 319 & 400 \\
\hline & 10.6 & 11.7 & 360 & 322 \\
\hline & 10.6 & 6.0 & 400 & 422 \\
\hline & 11.7 & 6.2 & 310 & 345 \\
\hline & 15.8 & 17 & 529 & 400 \\
\hline & 4 & 11 & 335 & 274 \\
\hline & 4.3 & 14 & 269 & 256 \\
\hline & 10 & 15 & 269 & 323 \\
\hline & 7 & 7 & 476 & 318 \\
\hline & 10 & 8 & 413 & 460 \\
\hline & 9 & 6 & 449 & 203 \\
\hline & 17 & 15 & 303 & 254 \\
\hline & 15 & 6 & 275 & 475 \\
\hline & 6 & 8 & 324 & 334 \\
\hline & 5 & 8 & 117 & 136 \\
\hline & 7 & 6 & 250 & 270 \\
\hline & 6 & 8 & 177 & 306 \\
\hline & 9 & 9 & 268 & 374 \\
\hline & 8 & 14 & 398 & 450 \\
\hline & 6 & 7 & 460 & 400 \\
\hline & 7 & 7 & 416 & 380 \\
\hline & 7 & 9 & 299 & 424 \\
\hline & 12 & 7 & 455 & 800 \\
\hline & 15 & 7 & 408 & 490 \\
\hline & 8 & 5 & 600 & 560 \\
\hline & 8 & 10 & 425 & 376 \\
\hline & 4 & 3 & 277 & 280 \\
\hline & 9 & 13 & 324 & 600 \\
\hline & 10 & 12 & 387 & 288 \\
\hline & 13 & 11 & 520 & 329 \\
\hline Ref range & \multicolumn{2}{|c|}{$3-1410^{9} / \mathrm{L}$} & \multicolumn{2}{|c|}{$150-450$} \\
\hline Mean & 9.8142 & 9.6172 & 358.32 & 369.4 \\
\hline Variance & 14.9 & 8.8 & 18257.9 & 15521.6 \\
\hline SD & 3.86 & 2.97 & 135.12 & 124.59 \\
\hline $\mathrm{P}(\mathrm{T}<=\mathrm{t}) 2$-tai $l$ & \multicolumn{2}{|c|}{0.747031} & \multicolumn{2}{|c|}{0.58563866} \\
\hline
\end{tabular}

$\mathrm{kg} /$ day). ${ }^{28,29}$ The recommended maintenance dose of VPA starts from $10-15 \mathrm{mg} / \mathrm{kg} /$ day up to $60 \mathrm{mg} / \mathrm{kg} /$ day. Some studies recommended up to $100 / \mathrm{mg} / \mathrm{kg} /$ day. ${ }^{30}$ Valproic acid average dose in this cohort was 31.4 $\mathrm{mg} / \mathrm{kg} /$ day which can be one factor that can explain its tolerability in this study.

Polytherapy is another reported factor contributing to valproic acid related major side effects. ${ }^{31}$ In our study, not all patients on polytherapy had side effects. However, reported side effects of encephalopathy and mild increase in LFT were seen in children receiving VPA and one or more ASMs. Most children with intractable epilepsy will need polytherapy. However, rational polytherapy is recommended. Medications like carbamazepine and phenytoin were reported to increase the level of VPA and hence its toxicity. ${ }^{32}$ Star et el showed that when VPA is used as monotherapy, less side effects were reported. ${ }^{33}$ Polytherapy and fatal side effects were (58\%) in VigiBase compared with non-fatal outcome $(34 \%){ }^{33}$

Some of commonly reported valproic acid side effects are: nausea, alopecia, diarrhea, vomiting, and increased appetite. Rare but possibly fatal side effects like pancreatitis and hepatotoxicity should be monitored especially in young children. However, fatalities due to VPA-induced encephalopathy and liver disease are not limited to children. It can occur across different age groups. Bryant et al reviewed US experience with VPA. Out of 7630 patients below the age of 2 years received valproic acid, 5 patients developed fatal hepatotoxicity $(0.06 \%) .^{7}$ There have been 268 individual case safety reports "ICSR" to the WHO Global ICSR database from 55 countries since $1977 .{ }^{31}$

Seizure frequency in most of patients improved (64\%) after introducing VPA. Despite a moderate dose of VPA $(31 \mathrm{mg} / \mathrm{kg} /$ day $)$, some of the children in this cohort were even seizure-free. Some studies reported cessation of infantile spasms in more than $70 \%$ of their cohort after introducing VPA. Valproic acid is one of the effective antiseizure medications in infantile epilepsies. 35,36 It is one of the first line medications for Dravet syndrome, myoclonic epilepsy and generalized epilepsy across different age groups. ${ }^{36}$

The limitations of the study are the retrospective design, and the relatively small sample size.

In conclusion, Valproic acid is an effective and tolerable ASM in this age group. It can be considered for the treatment of seizures in children below the age of 2 years. It is potentially safer when used in moderate doses with close follow up of LFT and valproic acid levels. Avoiding polytherapy and concomitant use of enzyme inducers is recommended. Screening for children with metabolic and hepatic diseases should be 
considered. More studies and a better understanding of VPA-related age pharmacokinetics and epigenetics are recommended.

Acknowledgement. The authors would like to thank Scribendi company (https://www.scribendi.com) for English language editing.

\section{References}

1. Hunter MB, Yoong M, Sumpter RE, Verity K, Shetty J, McLellan A, et al. Incidence of early-onset epilepsy: A prospective population-based study. Seizure 2020; 75: 49-54.

2. Bombardieri R, Pinci M, Moavero R, Cerminara C, Curatolo P. Early control of seizures improves long-term outcome in children with tuberous sclerosis complex. Eur J Paediatr Neurol 2010; 14: 146-149.

3. Sahu PK, Mishra D, Juneja M, Taneja K. Clinico-etiological Profile and Developmental Status of Infants Aged 1-24 months with Epilepsy. Indian J Pediatr 2019; 86: 681-685.

4. Eltze CM, Chong WK, Cox T, Whitney A, Cortina-Borja M, Chin RF, et al. A population-based study of newly diagnosed epilepsy in infants. Epilepsia 2013; 54: 437-445.

5. Kwon SH, Scheinost D, Lacadie C, Benjamin J, Myers EH, Qiu $\mathrm{M}$, et al. GABA, resting-state connectivity and the developing brain. Neonatology 2014; 106: 149-155.

6. Tomson T, Battino D, Perucca E. Valproic acid after five decades of use in epilepsy: time to reconsider the indications of a time-honoured drug. Lancet Neurol 2016; 15: 210-218.

7. Bryant AE 3rd, Dreifuss FE. Valproic acid hepatic fatalities. III. U.S. experience since 1986. Neurology 1996; 46: 465-469.

8. Wang Y, Hu W, Li Z. Influence of age and co-medication on the concentration and efficacy of valproic acid in Chinese epilepsy children. Pak J Pharm Sci 2020; 33: 537-542.

9. Koenig SA, Buesing D, Longin E, Oehring R, Häussermann P, Kluger G, et al. Valproic acid-induced hepatopathy: nine new fatalities in Germany from 1994 to 2003. Epilepsia 2006; 47: 2027-2031.

10. Ghodke-Puranik Y, Thorn CF, Lamba JK, Leeder JS, Song W, Birnbaum AK, et al. Valproic acid pathway: pharmacokinetics and pharmacodynamics. Pharmacogenet Genomics 2013; 23 : 236-241.

11. Zhu MM, Li HL, Shi LH, Chen XP, Luo J, Zhang ZL. The pharmacogenomics of valproic acid. J Hum Genet 2017; 62: 1009-1014.

12. Guo HL, Jing X, Sun JY, Hu YH, Xu ZJ, Ni MM, et al. Valproic Acid and the Liver Injury in Patients with Epilepsy: An Update. Curr Pharm Des 2019; 25: 343-351.

13. Meseguer ES, Elizalde MU, Borobia AM, Ramírez E. Valproic Acid-Induced Liver Injury: A Case-Control Study from a Prospective Pharmacovigilance Program in a Tertiary Hospital. J Clin Med 2021; 10: 1153.

14. Cofini M, Quadrozzi F, Favoriti P, Favoriti M, Cofini G. Valproic acid-induced acute pancreatitis in pediatric age: case series and review of literature. G Chir 2015; 36: 158-160.

15. Rosati A, Ilvento L, Lucenteforte E, Pugi A, Crescioli G, McGreevy KS, et al. Comparative efficacy of antiepileptic drugs in children and adolescents: A network meta-analysis. Epilepsia 2018; 59: 297-314.
16. Yang X, Wang P, Pan J, Wang C, Luo J, Ma H. Valproic acid concentration and biochemical indexes in epilepsy outpatients during monotherapy or combination therapy. Zhong Nan Da Xue Xue Bao Yi Xue Ban 2020; 45: 782-789.

17. Du Y, Lin J, Shen J, Ding S, Ye M, Wang L, et al. Adverse drug reactions associated with six commonly used antiepileptic drugs in southern China from 2003 to 2015. BMC Pharmacol Toxicol 2019; 20: 7.

18. Wilmshurst JM, Gaillard WD, Vinayan KP, Tsuchida TN, Plouin P, Van Bogaert P, Carrizosa J, Elia M, Craiu D, Jovic NJ, Nordli D, Hirtz D, Wong V, Glauser T, Mizrahi EM, Cross JH. Summary of recommendations for the management of infantile seizures: Task Force Report for the ILAE Commission of Pediatrics. Epilepsia 2015; 56: 1185-1197.

19. Ee LC, Shepherd RW, Cleghorn GJ, Lewindon PJ, Fawcett J, Strong RW, Lynch SV. Acute liver failure in children: A regional experience. J Paediatr Child Health 2003; 39: 107-110.

20. Hussein RS, Soliman RH, Abdelhaleem A, Tawfeik MH, Abdelrahim M. Effect of antiepileptic drugs on liver enzymes. Beni-Suef University Journal of Basic and Applied Sciences 2013: 2: 14-19.

21. Adedapo ADA, Demaki WE, Lagunju I. Non-Dose-Dependent Changes in Liver Enzyme Levels of Children With Epilepsy on Treatment With Sodium Valproate. Dose Response 2020; 18 : 1559325820918445.

22. Aithal GP, Watkins PB, Andrade RJ, Larrey D, Molokhia M, Takikawa H, Hunt CM, Wilke RA, Avigan M, Kaplowitz $\mathrm{N}$, Bjornsson E, Daly AK. Case definition and phenotype standardization in drug-induced liver injury. Clin Pharmacol Ther 2011; 89: 806-815.

23. Yu YC, Mao YM, Chen CW, Chen JJ, Chen J, Cong WM, et al. Drug-induced Liver Injury (DILI) Study Group; Chinese Society of Hepatology (CSH); Chinese Medical Association (CMA). CSH guidelines for the diagnosis and treatment of drug-induced liver injury. Hepatol Int 2017; 11: 221-241.

24. Chicharro AV, de Marinis AJ, Kanner AM. The measurement of ammonia blood levels in patients taking valproic acid: looking for problems where they do not exist? Epilepsy Behav 2007; 11: 361-366.

25. Gayam V, Mandal AK, Khalid M, Shrestha B, Garlapati P, Khalid M. Valproic acid induced acute liver injury resulting in hepatic encephalopathy- a case report and literature review. $J$ Community Hosp Intern Med Perspect. 2018; 8: 311-314.

26. Kriel RL, Fischer JH, Cloyd JC, Green KH, Fraser GL. Valproic acid pharmacokinetics in children: III. Very high dosage requirements. Pediatr Neurol 1986; 2: 202-208.

27. Ghozzi H, Hakim A, Sahnoun Z, Ben Mahmoud L, Atheymen R, Hammami S, Zeghal K. [Relationship between plasma concentrations of valproic acid and hepatotoxicity in patients receiving high doses]. Rev Neurol (Paris) 2011; 167: 600-606.

28. Serragui S, Zalagh F, Tanani DS, Ouammi L, Moussa LA, Badrane $\mathrm{N}$, et al. [Therapeutic drug monitoring of three antiepileptic drugs - Back on twenty years of experience]. Pan Afr Med J 2016; 25: 10.

29. Wahba A, Bergez E. Severe Pancytopenia Induced by Valproic Acid. Cureus 2020; 12: e11252.

30. Sirven JI, Noe K, Hoerth M, Drazkowski J. Antiepileptic drugs 2012: recent advances and trends. Mayo Clin Proc 2012; 87: 879-889. 
31. Nanau RM, Neuman MG. Adverse drug reactions induced by valproic acid. Clin Biochem 2013; 46: 1323-1338.

32. Koenig SA, Buesing D, Longin E, Oehring R, Häussermann P, Kluger G, et al. Valproic acid-induced hepatopathy: nine new fatalities in Germany from 1994 to 2003. Epilepsia 2006; 47: 2027-2031.

33. Star K, Edwards IR, Choonara I. Valproic acid and fatalities in children: a review of individual case safety reports in VigiBase. PLoS One 2014; 9: e108970.
34. Auvichayapat N, Tassniyom S, Treerotphon S, Auvichayapat P. Treatment of infantile spasms with sodium valproate followed by benzodiazepines. J Med Assoc Thai 2007; 90: 1809-1814.

35. Siemes H, Spohr HL, Michael T, Nau H. Therapy of infantile spasms with valproate: results of a prospective study. Epilepsia 1988; 29: 553-560.

36. Wilmshurst JM, Burman R, Gaillard WD, Cross JH. Treatment of infants with epilepsy: Common practices around the world. Epilepsia 2015; 56: 1033-1046.

\section{Illustrations, Figures, Photographs}

All figures or photographs should be submitted in a high resolution (minimum 300 DPI) electronic version saved in jpeg or tiff format. Original hard copies of all figures may be requested when necessary. Photographs will be accepted at the discretion of the Editorial Board. All lettering, arrows, or other artwork must be done by an artist or draftsman. If arrows are used please ensure they appear in a different color to the background color, preferably black with a white border, or white with a black border. If arrows distinguish different items on the figure then different arrow styles should be used ie. long, short, wide, narrow. Written informed consent for publication must accompany any photograph in which the subject can be identified. Written copyright permission, from the publishers, must accompany any illustration that has been previously published. 